\title{
Weitere Themen
}

Stefan Altekamp

\section{Der Umbruch der Bibliothekslandschaft in SBZ und früher DDR im Spiegel einer kleinen Spezialbibliothek Radical Changes in the Library Landscape of the Soviet Occupation Zone and Early GDR from the Angle of a Small Special Library}

https://doi.org/10.1515/bd-2020-0047

\begin{abstract}
Der Beitrag analysiert die konkreten Auswirkungen von Entnazifizierung, Umverteilung von Bibliotheksgut, Bezugsmöglichkeiten im Antiquariatsbuchhandel und Angeboten der Buchreproduktion in Fotografie und Mikroformen auf die Bestandsgeschichte einer Spezialbibliothek vor dem Hintergrund des Umbruchs der Bibliothekslandschaft in der Sowjetischen Besatzungszone und der frühen DDR.
\end{abstract}

Schlüsselwörter: Provenienz, Antiquariatsbuchhandel, Entnazifizierung

\begin{abstract}
Our contribution analyses the practical effects of denazification, redistribution of library assets and materials, purchase options in the antiquarian book trade sector and library services such as the reproduction of books in photographic and microfiche forms, as mirrored in the history of a specialised library against the backdrop of radical changes in the library landscape of the Soviet Occupation Zone and during the early days of the German Democratic Republic.
\end{abstract}

Keywords: Provenance research, antiquarian book trade, denazification 
Im Folgenden wird der Bestandsaufbau einer kleinen universitären Bibliothek vor seinem zeit- und bibliotheksgeschichtlichen Hintergrund der 1940er und 1950er Jahre betrachtet ${ }^{1}$.

Die Fachbibliothek Klassische Archäologie der Humboldt-Universität zu Berlin hat seit 1851 die Entwicklung von einer für den akademischen Unterricht griffbereit vorgehaltenen Handbibliothek (im Rahmen eines „Archäologischen Apparats“) über eine veritable Spezialbibliothek („Institutsbibliothek“) hin zum Aufbauelement eines einschichtigen universitären Bibliothekssystems (,Zweigbibliothek“) durchlaufen. 1944 wurden aus dem damals etwa 10.000 Bände umfassenden Bestand etwa 7.000 Bände Richtung Westen aus Berlin ausgelagert, um sie vor drohender Vernichtung im Bombenkrieg zu schützen. Nach längerer Odyssee wurde diese in 144 Kisten verpackte Teilbibliothek der Freien Universität in Berlin-West zur Verfügung gestellt. In der Rumpfbibliothek in Berlin-Ost wurde ab 1946 ein energischer Neuaufbau mit dem Ziel einer weitgehenden Rekonstruktion des Altbestandes betrieben. Die Ersatzbeschaffungen sind auch ein materieller Abdruck der massiven Umbrüche der zeitgenössischen Bibliothekslandschaft mit ihren Auswirkungen auf Institutionen und Mechanismen der Buchverteilung. Sie sind zudem Produkt einer dirigistischen Ökonomie, die sowohl außergewöhnliche Optionen eröffnete wie enervierende Zwänge erzeugte. Die Zahl der konkreten Belege aus dieser kleinen Bibliothek für jeden einzelnen Vorgang ist gering, jedoch handelt es sich gewiss um typische Fälle, die hier in einen Gesamtkontext gestellt werden sollen.

War der Ausgleich von Verlagerungsverlusten ein zentrales Anliegen der fachlichen Einrichtung (,Winckelmann-Institut“), so galt es parallel mit den Anforderungen einer Bestandsbereinigung umzugehen. Entnazifizierung von Buchbeständen war in der Sowjetischen Besatzungszone (SBZ), später in der DDR, einerseits ein hochoffizielles Programm, andererseits - in der Theorie - ein formal streng reglementiertes Procedere ${ }^{2}$. Richtschnur für Aussonderungen war

1 Ausführlich zur Bibliotheks- und Bestandsgeschichte mit umfangreicher Bilddokumentation: Altekamp, Stefan; Weiße, Antonia (Hg.): Bibliotheksarchäologie. Zur Geschichte der Zweigbibliothek Klassische Archäologie der Humboldt-Universität zu Berlin. Berlin 2016, http://edoc. hu-berlin.de/18452/14322 [Zugriff: 12.02.2020].

2 Greguletz, Alexander: Literarische Säuberungen und ihre politischen Metamorphosen im Bibliothekswesen der DDR 1945 bis 1949. In: Buch und Bibliothek 42 (1990), S. 534-538; Steigers, Ute: Die Mitwirkung der Deutschen Bücherei an der Erarbeitung der „Liste der auszusondernden Literatur" in den Jahren 1945 bis 1951. In: Zeitschrift für Bibliothekswesen und Bibliographie 38 (1991), S. 236-256; Amedick, Sigrid: „Macht die wissenschaftlichen Bibliotheken zu sozialistischen Einrichtungen!“. Bibliotheken, Bibliothekare und Politik in der SBZ und DDR 1945 bis 1965. In: Bibliothek und Wissenschaft 31 (1998), S. 62-73; Hamann, Olaf: Faschistische Literatur in deutschen Bibliotheken. Über Aussonderungen und Neuorientierungen im Bestandsaufbau 
insbesondere die „Leipziger Liste“, die in vier zwischen 1946 und 1953 erschienenen Bänden explizit diejenigen Veröffentlichungen aufführte, die aus allen Bibliotheken zu entfernen waren ${ }^{3}$. In dieser Liste sollten auch sämtliche Fachpublikationen erfasst sein, die inhaltlich Positionen der nationalsozialistischen Ideologie widerspiegelten. Mit dieser Zielsetzung standen die Bibliothekare, die die Liste zusammengestellt haben, angesichts disziplinärer Spezialisierung und Aufsplitterung aber vor einer unlösbaren Aufgabe. Daher zog die Kommission gerne eher formale Kriterien heran (z. B. Indizierung ganzer Reihen mit Reizwörtern in den Reihentiteln). Grundsätzlich sind ihr durchaus weit verbreitete z. B. altertumswissenschaftliche Werke, die stark von NS-Gedanken infiziert waren, entgangen. So ist aus dem einschlägigen Oeuvre Fritz Schachermeyrs lediglich dessen „Lebensgesetzlichkeit in der Geschichte“ (1940) aufgefallen ${ }^{4}$, von den Publikationen Joseph Vogts ausgerechnet und ausschließlich „Cicero und Sallust über die Catilinarische Verschwörung“ (1938) ${ }^{5}$. Das zuletzt genannte Buch Vogts war allerdings in der als Ganzes inkriminierten Reihe „Auf dem Wege zum nationalpolitischen Gymnasium“ erschienen. Insgesamt unbehandelt blieb das Problem der Zeitschriftenliteratur, denn eindeutig im Sinne der „Leipziger Liste“ zu verstehende Texte konnten sich leicht zwischen den Buchdeckeln ansonsten unauffälliger Fachperiodika oder Sammelbände verbergen. Das trifft z. B. auf krass rassistische Aufsätze des Klassischen Archäologen Reinhard Herbig zu, dessen Name in der Liste nicht auftaucht ${ }^{6}$.

Die Bibliotheken allerdings trugen nicht nur das Risiko der Sanktion, wenn sie die Listen nicht abarbeiteten, sondern hatten auch der Forderung Rechnung

wissenschaftlicher Bibliotheken in der Zeit 1945-1949 am Beispiel der Öffentlichen Wissenschaftlichen Bibliothek Berlin (ÖWiBi). In: Heukenkamp, Ursula (Hg.): Schuld und Sühne? Kriegserlebnis und Kriegsdeutung in deutschen Medien der Nachkriegszeit (1945-1961). Amsterdam 2001, S. 525-540; Hammer, Angela: Aussonderung nationalsozialistischer Literatur in ostdeutschen Bibliotheken nach dem Zweiten Weltkrieg am Beispiel der Universitätsbibliothek der Humboldt-Universität zu Berlin. In: Bibliothek. Forschung und Praxis 37 (2013), S. 331-346. 3 Deutsche Verwaltung für Volksbildung in der sowjetischen Besatzungszone / Ministerium für Volksbildung der Deutschen Demokratischen Republik: Liste der auszusondernden Literatur. Berlin 1946.1947.1948. 1953 - im Folgenden „Leipziger Liste“, http://www.polunbi.de/bibliothek/ 1946-nslit.html, http://www.polunbi.de/bibliothek/1947-nslit.html, http://www.polunbi.de/bib liothek/1948-nslit.html, http://www.polunbi.de/bibliothek/1953-nslit.html [Zugriffe: 12.02.2020]. 4 Leipziger Liste 1948 wie Anm. 3, S. 248.

5 Leipziger Liste 1946 wie Anm. 3, S. 429.

6 Herbig, Reinhard: Herakles im Orient. Heroenglaube und Geschichtserlebnis. In: Corolla. Ludwig Curtius zum sechzigsten Geburtstag dargebracht. Stuttgart 1937, S. 205-211; ders.: Philister und Dorier. In: Jahrbuch des Deutschen Archäologischen Instituts 55 (1940), S. 58-89; ders.: Das archäologische Bild des Puniertums. In: Vogt, Joseph (Hg.): Rom und Karthago. Ein Gemeinschaftswerk. Leipzig 1943, S. 139-177. 
zu tragen, dass nicht nur nach den Buchstaben, sondern auch nach dem Geiste des Index zu verfahren war. D. h. auch solche Werke waren betroffen, die zwar nicht auf den Listen standen, aber inhaltlich mit den gelisteten gleichzusetzen waren ${ }^{7}$.

Für Berliner Bibliotheken kam erschwerend hinzu, dass ein „Säuberungsausschuß für das Büchereiwesen im Auftrag der Abteilung für Volksbildung im Magistrat der Stadt Berlin“ 1946 ein eigenes Berliner „Verzeichnis“ erstellt hatte ${ }^{8}$. Dieses noch radikalere Instrument, an dem auch die ehemalige Preußische Staatsbibliothek, nun Öffentlich-Wissenschaftliche Bibliothek (ÖWB) und die Universitätsbibliothek mitzuwirken hatten ${ }^{9}$, verstand sich ausdrücklich nur als eine Beispielliste. Auch solche Bestände waren auszusondern, die den konkret gelisteten inhaltlich entsprachen ${ }^{10}$. Die Ausweitung des Selektionsauftrags musste in diesem Falle geradezu geboten erscheinen, erweist sich das „Verzeichnis“ doch angesichts des frühen Erstellungsdatums und des relativ knappen Umfangs als im Sinne seiner Intentionen sehr lückenhaft. So indizierte es zwar den „NS-Rasseforscher“ Hans F.K. Günther mit seinem gesamten Oeuvre ${ }^{11}$, übersah aber den ebenso prominenten Eugen Fischer. Auf der anderen Seite setzte die Liste z. T. bizarre Akzente. Dem pauschalen Bann unterlagen auch Karl Friedrich Schinkel als „völkisch“12 oder Felix Dahn als „völkischer Pathetiker“ bzw. „teutonischer

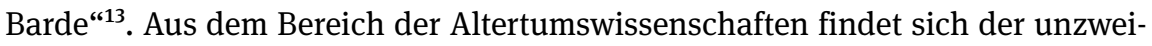
felhaft NS-affine Althistoriker Franz Altheim mit vier Publikationen ${ }^{14}$.

Das „Verzeichnis“ stellte auch insofern extreme Anforderungen, als das pauschalisierende Aussonderungsgebot äußerst weit griff, indem es sogar ästhetische Kriterien heranzog, auf der anderen Seite aber grundsätzlich beanstandete Werke als historische Quellen bewahrt, wenn auch der freien Nutzung entzogen werden sollten $^{15}$. Hier finden sich z. B. sämtliche Schriften Kaiser Wilhelms II. angegeben, der u.a. in der Archäologie publizistisch dilettiert hatte ${ }^{16}$.

Die Bibliotheken sind auf sehr unterschiedliche Weise mit diesen Herausforderungen umgegangen: In Berlin haben sich große Bibliotheken wie die ÖWB und

7 Leipziger Liste 1946 wie Anm. 3, S. 4.

8 Abteilung für Volksbildung im Magistrat der Stadt Berlin: Verzeichnis der auszusondernden Literatur. Berlin 1946.

9 Hamann wie Anm. 2, S. 527 f.

10 Verzeichnis wie Anm. 8, S. 3.

11 Verzeichnis wie Anm. 8, S. 34.

12 Verzeichnis wie Anm. 8, S. 67.

13 Verzeichnis wie Anm. 8, S. 5, 20.

14 Verzeichnis wie Anm. 8, S. 87.

15 Verzeichnis wie Anm. 8, S. $179 \mathrm{f}$.

16 Verzeichnis wie Anm. 8, S. 183. 
die Universitätsbibliothek recht strikt an die Vorschriften gehalten. Ihr Vorgehen wurde von der Sowjetischen Militäradministration überwacht ${ }^{17}$. Die Verantwortlichen im Winckelmann-Institut dagegen begegneten den Schwierigkeiten der Auslegung der „Listen“ damit, dass sie das Entnazifizierungsprogramm völlig ignorierten. Während die Theorie Radikalität verlangte und das reale Ausmaß der Aussonderungen und Separierungen insgesamt den Eindruck rigoroser Konsequenz erzeugte, lässt die Bestandsentwicklung der kleinen hier betrachteten Bibliothek Zweifel an der faktischen Durchschlagskraft der Maßnahmen aufkommen (s. u.). Im Winckelmann-Institut erfolgte lediglich eine wahrscheinlich zentral angeordnete kosmetische „Entnazifizierung“ mit der Überstempelung aller Hoheitszeichen auf älteren Stempeln - vom preußischen Adler (auch in der Weimarer Version) bis zum Hakenkreuz.

Rein praktisch hätten auszusondernde Bestände an die ÖWB (nachmalige Deutsche Staatsbibliothek) übergeben werden müssen, die ein entsprechendes Auffangmagazin für alle wissenschaftlichen Bibliotheken im Norden der SBZ bzw. DDR aufbaute, für den sich der Name „Sperrbibliothek“ einbürgerte ${ }^{18}$. Mit Auflösung der später offiziell als „Abteilung für spezielle Forschungsliteratur“ (ASF) firmierenden Schattenbibliothek, die bis 1989 z.B. auch zensierte Westliteratur aufnahm, wurden die aus anderen Bibliotheken übernommenen Bestände rückübertragen, so auch 1991-93 an die Universitätsbibliothek der Humboldt-Universität ${ }^{19}$. In der Bibliothek im Winckelmann-Institut, in der ja trotz Auslagerung ein Rumpfbestand von etwa 3.000 Bänden verblieben war, sind offenkundig keine Anstalten zur Abgabe belasteter Literatur an die „Sperrbibliothek“ gemacht worden. Erst acht Jahre nach Kriegsende veranlasste eine Revision in den Institutsbibliotheken die Universitätsleitung $\mathrm{zu}$ der Verfügung, noch verbliebene NS-Literatur endgültig auszuliefern: Dem Winckelmann-Institut quittierte die „Sperrbibliothek“ am 23. Februar 1953 die Übernahme folgender Veröffentlichungen $^{20}$ :

17 Hamann wie Anm. 2, S. 530. - Nach Hammer wie Anm. 2, S. 339 fand keine systematische Kontrolle durch die SMAD statt, allerdings standen die Bibliotheken unter der Sozialkontrolle ihrer Nutzer.

18 Schubarth, Karl: Die Sperrbibliothek. In: Das Stichwort. Hauszeitschrift der Staatsbibliothek zu Berlin 35.4 (1991), S. 40-42; Amedick wie Anm. 2, S. 117-122; Waligora, Raimund: Der Giftschrank der Staatsbibliothek zu Berlin. In: Lokatis, Siegfried; Sonntag, Ingrid (Hg.): Heimliche Leser in der DDR. Kontrolle und Verbreitung unerlaubter Literatur. Berlin 2008, S. 191-200; Hammer wie Anm. 2, S. 341-343.

19 Hammer wie Anm. 2, S. 344.

20 Winckelmann-Institut der Humboldt-Universität: Aktenordner „Bibliothek: Revisionen, Inventur, Statistik“. - Erlass der Universitätsleitung: Hammer wie Anm. 2, S. 340 f. 
1. Zeitschrift für Rassenkunde 1935-39 (5 Bände)

2. Günther, Rassengeschichte des hellenischen und des römischen Volkes

3. Reche, Rasse und Heimat der Indogermanen

4. Günther, Rassenkunde des jüdischen Volkes

5. Günther, Rassengeschichte des hellenischen und des römischen Volkes [sic]

6. Günther, Rasse und Stil

7. Günther, Die Nordische Rasse bei den Indogermanen Asiens

8. Fischer/Kittel, Das antike Weltjudentum

9. Wilhelm II., Erinnerungen an Korfu ${ }^{21}$

10. Sieglin, Die blonden Haare der indogermanischen Völker des Altertums

Einige zentrale NS-Propagandaschriften (v. a. Hitler, „Mein Kampf“; Hitler-Reden), die aus offenkundig opportunistischen Gründen zwischen 1934 und 1940 für die Bibliothek angekauft worden waren, wurden wohl aus ebenso nachvollziehbar opportunistischen Motiven nach Kriegsende stillschweigend aussortiert, ohne weitere administrative Spuren zu hinterlassen. Im Unterschied $\mathrm{zu}$ dieser Politliteratur galten die 1953 auf Druck an die „Sperrbibliothek“ abgegebenen Publikationen bei ihrer Anschaffung als fachnah. Es fällt auf, dass dezidiert rassistische Literatur - auch bei länger zurückliegendem Erscheinungsdatum - in einer gewissen Geschlossenheit 1938 oder kurz darauf angeschafft worden ist. Im Wintersemester 1939/40 hatte der Lehrstuhlinhaber Gerhart Rodenwaldt eine Lehrveranstaltung zum Thema „Kunst, Rasse und Volkstum in der Antike“ angeboten $^{22}$. Fachliche Relevanz scheint den unterschiedslos pseudowissenschaftlichen Werken ungeachtet der offiziellen Entnazifizierungsschlacht auch nach 1945 zugestanden worden zu sein.

Im Anschluss an die Auflösung der „Sperrbibliothek“ im Gefolge der Friedlichen Revolution wurden ebenfalls die aus den Institutsbibliotheken übernommenen Bestände restituiert ${ }^{23}$. Auch ein kleines Kontingent aus der Klassischen Archäologie - nicht alle Bände der 1953er Liste konnten identifiziert werden kehrte in die Universität zurück; es verblieb in der Universitätsbibliothek.

Nur spärliche Hinweise finden sich für eine mögliche Separierung bestimmter Werke im Winckelmann-Institut selber. Der 1943 von Joseph Vogt herausgegebene Sammelband „Rom und Karthago“, der sich zu einer aktualistischen „rassegeschichtlichen“ Interpretation der Rom-Karthago-Konflikte aufschwang, wurde

21 Werke von und über Wilhelm II. waren im Berliner „Verzeichnis“ unter der Kategorie B gelistet: Verzeichnis wie Anm. 8, S. 183.

22 Altekamp, Stefan: Klassische Archäologie und Nationalsozialismus. Berlin 2016, S. 131-133, http://edoc.hu-berlin.de/18452/14308 [Zugriff: 12.02.2020].

23 Hammer wie Anm. 2, S. $344 \mathrm{f}$. 
laut Vermerk im Karten-Standortkatalog in einem „Giftschrank“ aufbewahrt. Auch Schriften des umstrittenen Archäologen Ernst Buschor sollen zeitweilig von dessen Schüler Ludger Alscher, der seit 1951 die Klassische Archäologie an der Humboldt-Universität vertrat, separiert worden sein, um sie aus der Schusslinie möglicher Kritik zu nehmen ${ }^{24}$. Im Karten-Standortkatalog findet sich mit Ausnahme der genannten Publikation Vogts kein weiterer Hinweis auf eine Separierung aus ideologischen Gründen. Das betrifft auch die Werke Buschors. Dessen „Das Kriegertum der Parthenonzeit“ von 1943, ein den Krieg als solchen transzendentierender Essay, der zynischerweise den Frontsoldaten zugesandt worden war, findet sich gleich zweimal im Bestand an. In einem der beiden Exemplare sind die martialischen Vorsprüche des Autors und des Dekans der Münchener Philosophischen Fakultät, Franz Dirlmeier („Soldaten, Soldaten!“) abgeklebt. Publikationen wie Erhart Kästners „Griechenland. Ein Buch aus dem Kriege“ von 1942 oder Fritz Schachermeyrs „Indogermanen und Orient“ von 1944 verblieben offensichtlich ohne Nutzungsbeschränkung im Freihandbestand.

Die nicht bzw. verspätet berücksichtigte Pflicht zur Abgabe indizierter Literatur ist allerdings nur eine Seite der Medaille. Auf der anderen steht der Umgang mit belasteter Literatur im Zuge der Ersatzbeschaffungen. In der Tat ist zu konstatieren, dass im Rahmen der versuchten Rekonstruktion der verlorenen Bibliotheksbestände auch NS-Literatur nachgekauft wurde. Fachgeschichtlich kann dabei diskutiert werden, ob diese Ergänzungspolitik als formalistische Pedanterie, quellenbewusste Bestandspflege oder ideologische Affinität zu deuten ist. Doch diese Bewertung steht hier nicht im Vordergrund. Bibliotheksgeschichtlich ist von Belang, dass die Nachbeschaffungen über offizielle Bezugswege ohne weiteres möglich gewesen sind.

Der oben erwähnte, von Joseph Vogt herausgegebene und vorübergehend in einem „Giftschrank“ aufbewahrte Sammelband von 1943 ist 1954 als Ersatzbeschaffung inventarisiert worden. Franz Altheims „Die Soldatenkaiser“ („Deutsches Ahnenerbe“, 1939) wurde 1949 angeschafft, 1952 und 1953 das zweibändige „Die Krise der Alten Welt“ desselben Autors. Dieses Werk war im Verzeichnis des Magistrats von Berlin von 1946 explizit als auszusondern aufgeführt gewesen ${ }^{25}$, Publikationen des „Ahnenerbe“-Verlages waren nach den Vorgaben des „Verzeichnisses“ insgesamt gebannt ${ }^{26}$. Buchstabe und Geist des „Verzeichnisses“ und der „Leipziger Liste“ haben demnach den antiquarischen Handel in der SBZ bzw. in der DDR nicht strikt gesteuert. Die offensichtlich unregelmäßige

24 Mündliche Auskunft Detlef Rößler.

25 Verzeichnis wie Anm. 8, S. 87.

26 Verzeichnis wie Anm. 8, S. 177. 
Befolgung der offiziellen Entnazifizierungsbefehle verweist erneut auf die Notwendigkeit einer Bibliotheksgeschichte „von unten“, die auf den materiellen Gegebenheiten der Bestände und den in diese eingeschriebenen Verwaltungsvorgängen fußt.

In mehreren Büchern in der Bibliothek im Winckelmann-Institut sind Teile des Inhaltes geschwärzt oder entfernt. Diese Bereinigungen erfolgten entweder durch den Vorbesitzer oder im Handel - die betreffenden Veröffentlichungen wurden erst nach 1945 erworben. Bislang fanden sich keine derartigen Eingriffe in Büchern, die vor 1945 in die Bibliothek gelangt waren:

- Franz Altheim, Die Krise der Alten Welt, Band 1, 1943 (Inv. 1953/439 - Kauf: Mehring, Leipzig): Der Verlagsname „Ahnenerbe“ ist überklebt, einzelne Seiten sind entfernt. In Band 2, der gesondert beschafft wurde (Inv. 1952/42 = 11.566; Kauf: Harrassowitz, Leipzig), ist der Verlagsname nicht unkenntlich gemacht worden, herausgetrennte Seiten finden sich aber auch hier.

- Thieme-Becker, Allgemeines Lexikon der bildenden Künstler, Band 35, 1942 (Inv. 1954/118 - Kauf: Debeg, Leipzig): Viele einzelne Formulierungen sind penibel geschwärzt, so die Bezeichnung „Jude“, „jüdische“ usw.

- Fritz Taeger, Hellas, 1940 (Inv. 1959/144 - Kauf: s.l., Otto Kruhl): Seiten wurden entfernt und Passagen geschwärzt.

- Richard Heinze, Die augusteische Kultur, 1939 (Inv. 1965/240 - Kauf: Zentralantiquariat, Leipzig): Passagen im Vorwort wurden überklebt.

- Konstantin A. Doxiades, Raumordnung im griechischen Städtebau, 1937 (Inv. 1977/37 - Kauf: Zentralantiquariat, Berlin): Das Vorwort mit Zitat eines „Führer“-Spruches wurde überklebt.

Die Publikation:

- Assien Bohmers, Die Aurignacgruppe etc., 1942 (Inv. 47/1955 - Kauf: Dorsch, Hagenow) war im „Ahnenerbe-Stiftung Verlag, Berlin-Dahlem“ erschienen, dessen Produktion als ganze eigentlich gebannt war (s. o.). Das Buch ist ohne Eingriffe wie Schwärzungen, Abklebungen usw. aus dem Handel übernommen worden.

Die Entfernung von NS-Emblemen bzw. die Schwärzung vereinzelter zu beanstandender Textpassagen hatte die Kommission der „Leipziger Liste“ verfügt. Diese Bereinigungen vorausgesetzt, sollten ansonsten inhaltlich unbeanstandete Bücher in den Beständen verbleiben können ${ }^{27}$. Skurril mutet die Entfernung (manchmal eher die Markierung) anrüchiger Namen und Zeichen an,

27 Leipziger Liste 1948 wie Anm. 3, S. 4. 


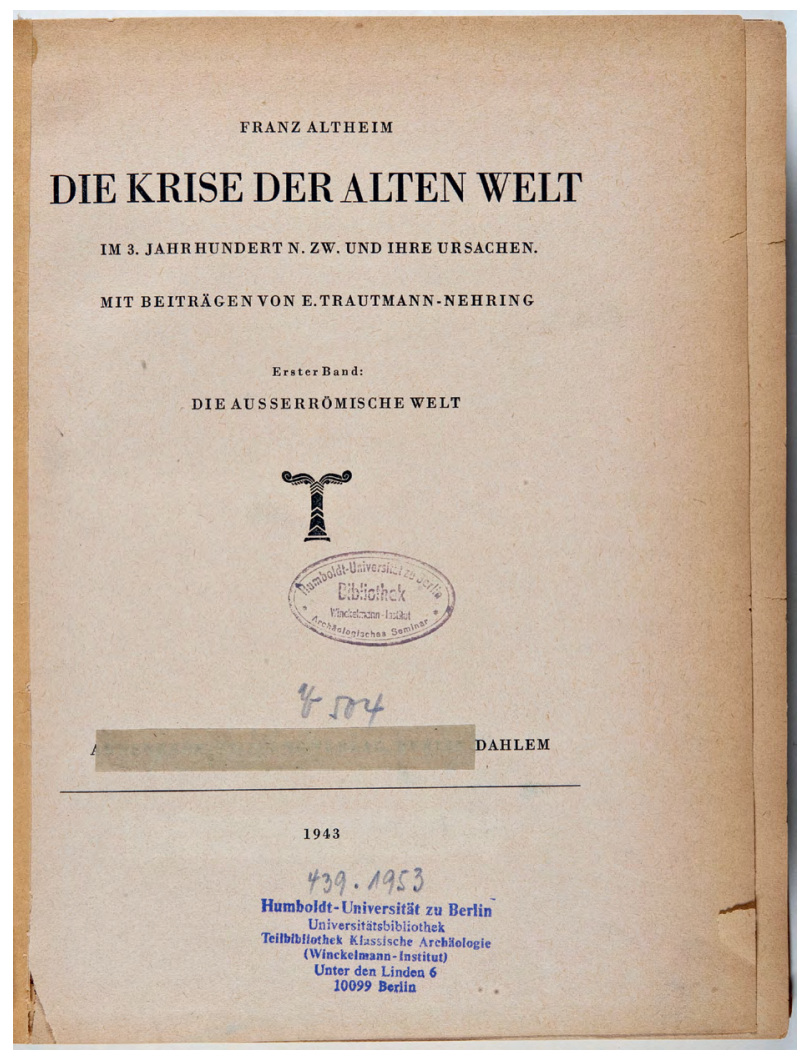

Abb. 1: Franz Altheim, Die Krise der Alten Welt (1943) mit überklebter Verlagsangabe „Ahnenerbe-Stiftung Verlag“, Foto: Antonia Weiße.

während die Inhalte unbeeinträchtigt zur Verfügung standen. Insgesamt zeigen die in der Bibliothek im Winckelmann-Institut nachvollziehbaren Erwerbungsprozesse eine relative Emanzipation des Handels gegenüber den rigiden Vorschriften.

Wenn die ursprünglich durch die „Leipziger Liste“ angestoßenen Bibliotheksmusterungen auf Unbedenklichkeit oder Gefährdung plädierten, konnte das wohl vor allem in Öffentlichen Bibliotheken - durch entsprechende Stempelungen kenntlich gemacht werden. Einige Bücher mit Vorbesitz tragen Prüfstempel, die anscheinend auf die fraglichen Bestandskontrollen zurückgehen:

- „Nicht für die Öffentlichkeit bestimmt. Empfänger ist für sachgemäße Verwendung verantwortlich“ (Johannes Bumüller, Leitfaden der Vorgeschichte Europas, 1925 - Inv. 1953/293 - Kauf: Mehring, Leipzig); 


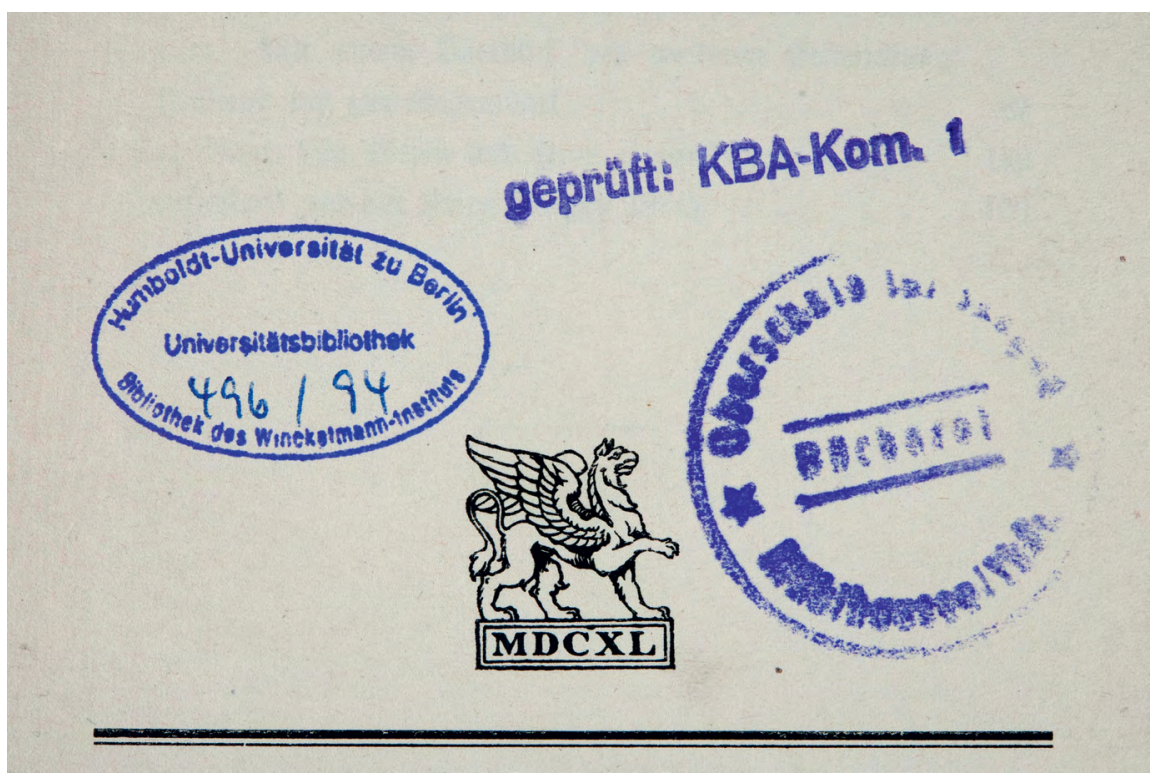

Abb. 2: Eduard Meyer, Weltgeschichte und Weltkrieg (1916) mit Prüfstempel „geprüft: KBA-Kom. 1“, Foto: Antonia Weiße.

- „Geprüft. Keine Beanstandungen. Kommission zur Säuberung der Büchereien Rostock“ (Joseph Gregor, Perikles, 1938 - Inv. 1961/59 - Kauf: Norddeutsches Antiquariat, Rostock);

- geprüft 19. Januar 1947“ (Flavius Arrianus, Anabasis Alexandri, 1910 - Inv. 1973/13 - Kauf: Zentralantiquariat, Leipzig);

- „Dieses Buch ist geprüft und kann in der Bücherei verbleiben“ (Eduard Norden, Die antike Kunstprosa, 1923 - Inv. 1973/98+99 - Kauf: Zentralantiquariat, Berlin);

- „geprüft: KBA-Kom. 1“ (Eduard Meyer, Weltgeschichte und Weltkrieg, 1916 Inv. 1994/496 - Geschenk aus Nachlass Wolfgang Schindler).

Die so unterschiedlichen Prüfstempel erinnern auch daran, dass die Entnazifizierung im engeren Sinne allmählich in eine politische wie ästhetische Zensur im weiteren Sinne übergegangen war. Der Stempel „Geprüft. Keine Beanstandungen. Kommission zur Säuberung der Büchereien etc." ist gut dokumentiert und in der Gemeinsamen Normdatei nachgewiesen ${ }^{28}$. Die Vielzahl der Stempel verweist auf

28 Körperschaftssatz GND: http://d-nb.info/gnd/107168020X [Zugriff: 12.02.2020]. 
die Vielfalt der Praxis. An die Arbeit einer Berliner „Bestandskommission“ erinnert sich Günter de Bruyn in seiner Autobiographie ${ }^{29}$.

Die Notwendigkeit massiver Rückergänzungen erhöhte die Bedeutung der antiquarischen Beschaffung, die vor dem Krieg eine deutlich geringere Rolle gespielt hatte. Der antiquarische Erwerb seinerseits spielte sich vor dem Hintergrund gewaltiger Buchumverteilungen in SBZ und früher DDR ab. Die Auflösung oder Verlagerung der Bestände von Kommunal-, Gymnasial-, Kirchen-, Privatoder sonstigen Bibliotheken setzte ungeheure Büchermengen frei, die in Teilen makuliert, in Teilen einer für diesen Zweck neu entstehenden Verteilungsbürokratie sowie dem expandierenden Antiquariatshandel überantwortet wurden.

1953, 1956 und 1957 übernahm die Bibliothek im Winckelmann-Institut insgesamt 18 ältere Publikationen mit Erscheinungsjahren zwischen 1808 und 1953 als „Geschenke“ von der 1953 eingerichteten „Zentralstelle für Wissenschaftliche Altliteratur“ (ZWA) in Gotha (ab 1959 in Berlin) ${ }^{30}$. Die ZWA war eine entscheidende Drehscheibe der Umverteilung von Büchern in der frühen DDR, da ihr die Aufgabe zufiel, unübersehbare Bestände aus aufgelassenen, beschlagnahmten oder verkleinerten Bibliotheken interessierten neuen Besitzern zuzuführen.

Von 12 im Bestand nach wie vor greifbaren Publikationen weisen sechs einen noch nachvollziehbaren Vorbesitz aus:

- Friedrich Hiller von Gaertringen, Inschriften von Priene, Berlin 1906 - Inv. G 4/1953 - Stempel: „Grossh. Regierungsbibliothek Schwerin“;

- Hugo Blümner, Die gewerbliche Thätigkeit der Völker des klassischen Alterthums, Leipzig 1869 - Inv. G 5/1953 - Stempel: „Der Rat der Stadt Dresden / Stadtbibliothek";

- Istituto Nazionale per le Relazioni Culturali con l'Estero, Bibliografie del Ventennio. Archeologia, Arti Figurative, Musica, Roma 1941 - Stempel 1: „Öff. Wissenschaftliche Bibliothek Berlin“ - Stempel 2: „Ausgeschieden Öff. Wiss. Bibl.“;

29 de Bruyn, Günter: Vierzig Jahre. Ein Lebensbericht. Frankfurt am Main 1996, S. 34 f. - Vgl. Knobloch, Heinz: Rund um das Buch. Leipzig 1973, S. 119.

30 Pachnicke, Gerhard: Die Arbeit an den wissenschaftlichen Altbeständen. In: Zentralblatt für Bibliothekswesen 68 (1954), S. 426-435; Tröger, Sigrid: Die Geschichte der Zentralstelle für wissenschaftliche Altliteratur. In: Zentralblatt für Bibliothekswesen 80 (1966), S. 415-425; Mälck, Andreas: Zum Wirken der Zentralstelle für wissenschaftliche Altbestände in Vergangenheit und Gegenwart. In: Zentralblatt für Bibliothekswesen 103 (1989), S. 537-545; Dehmel, Regine; Neumann, Hannah; Scheibe, Michaela: Akten der Deutschen Staatsbibliothek. Der Aktenbestand Zentralstelle für Wissenschaftliche Altbestände 1949-1995. Berlin 2019, S. 3-7, https:// provenienz.gbv.de/images/b/b8/SBB-PK_Akten_DSB_ZwA.pdf [Zugriff: 12.02.2020]. - Vgl. Ein Akt der grossen Kulturbarbarei. Die systematische Zerschlagung historischer Buchbestände in der DDR. In: Neue Zürcher Zeitung, 15. April 2002. 
- Friedrich Preller, Figuren-Fries zur Odyssee, Leipzig 1875 - Inv. G 15/1956 - Ex Libris: „Auguste Victoria Kaiserin u. Königin“;

- Christian Gottlob Heyne, Akademische Vorlesungen über die Archäologie der Kunst des Alterthums etc., Braunschweig 1822 - Inv. G 17/1953 - Stempel: „Gymnasium Zeitz“;

- Wilhelm Lübke, Geschichte der Plastik von den ältesten Zeiten bis auf die Gegenwart, Leipzig 1863 - Inv. G 20/1956 - Stempel: „Bibliothek des Königl. Dom-Gymnasiums zu Magdeburg“.

Über ihre Gothaer Anfangsjahre hinaus hat die ZWA allerdings nicht mehr an Kleinbibliotheken abgegeben. Diesen Einrichtungen blieb somit der sonstige Antiquariatsbuchhandel als Adressat für Ergänzungswünsche - wobei die ZWA auch diesen Handel (und damit auch den Export) bediente. Über Antiquariate gelangte eine größere Anzahl an Büchern aus weit gestreutem und disparatem Vorbesitz in die Bibliothek. Das Angebot aus den Antiquariaten speiste sich einerseits aus gebrauchten Büchern herkömmlicher Provenienz (Privatverkäufe, reguläre Bibliotheksaussonderungen, Dubletten), zum anderen aber aus zwangsweise aufgelösten oder dezimierten Sammlungen. Bücher aus Traditionsbibliotheken z. B. Schulbibliotheken - sind als verstreute Lückenfüller im Winckelmann-Institut nachweisbar ${ }^{31}$.

Trotz Unmengen an „freigesetzter“ Literatur konnte der Antiquariatsbuchhandel die fachspezifischen Rückergänzungswünsche der Klassischen Archäologie nur zu einem Teil erfüllen. Auf der anderen Seite war auch die neue im Westen erschienene Fachliteratur in der frühen DDR kaum zu beschaffen. Seit 1950 versprach ein alternatives Instrument der Bestandsergänzung doppelte Abhilfe: Der an der Staatsbibliothek („Öffentlich-Wissenschaftliche Bibliothek“) eingerichteten „Zentralstelle für Wissenschaftliche Literatur“ (ZWL) war die Aufgabe zugewiesen, die Versorgung mit wissenschaftlicher Literatur, die nicht in der DDR erschien, effektiv zu unterstützen ${ }^{32}$. Diesem Zweck diente zum einen

31 Siehe die Bilddokumentation „Vorbesitz“ in: Bibliotheksarchäologie wie Anm. 1, S. 176-215. 32 R., Zentralstelle für Wissenschaftliche Literatur. In: Neues Deutschland, 25. August 1950; Die Zentralstelle für Wissenschaftliche Literatur, ein Instrument des Fünfjahresplans. Berlin 1952; Götz, Wilhelm: Fünf Jahre Zentralstelle für Wissenschaftliche Literatur. 3. Aufl. Berlin 1957; Kunze, Horst: Grundzüge der Bibliothekslehre. 4. Aufl. Leipzig 1976, S. 385 f.; Umlauff, Ernst: Der Wiederaufbau des Buchhandels. Beiträge zur Geschichte des Büchermarktes in Westdeutschland nach 1945. Frankfurt am Main 1978, Sp. 1243-1246; Schaper, Christiane: Von der Zentralstelle für Literatur zum Institut für Dokumentation. Notwendigkeit, Schwierigkeiten, Nutzen. Diplomarbeit Humboldt-Universität zu Berlin, Institut für Bibliothekswissenschaft und Wissenschaftliche Information. Berlin 1990 (non vidi; von der Universitätsbibliothek der Humboldt-Universität makuliert). 
eine systematische Dokumentation, zum anderen der Betrieb eines Großlabors zur Reproduktion jener Publikationen, „die der Buchhandel nicht zu liefern vermag“33. Faktisch jedoch reproduzierten die 80 Mitarbeiter von Ermittlungsstelle und Fotolabor der ZWL ${ }^{34}$ ungeachtet der Lieferbarkeit der Vorlage im Handel. Die beachtliche Kapazität des Labors - es lieferte Anfang 1954 ca. 90.000 Fotorepros im Monat aus ${ }^{35}$ - erklärt sich vermutlich auch mit einer interessanten Kontinuität: Von 1920 bis 1945 arbeitete in der Preußischen Akademie der Wissenschaften (also in den Räumen der Staatsbibliothek) die „Reichsstelle für Naturwissenschaftliche (ab 1930: Wissenschaftliche) Berichterstattung“ (RWB), der ebenfalls die Aufgabe zugefallen war, Mängel der Literaturversorgung der deutschen Wissenschaft und Industrie auszugleichen. ${ }^{36}$ Dazu kam seit 1922 ein massenhaft betriebenes „Photokopieverfahren“ (eine fotografische Reproduktion) zum Einsatz ${ }^{37}$. Ursprünglich als Maßnahme gegen den Boykott der deutschen Forschung nach dem Ersten Weltkrieg gedacht, erreichte die Reproduktionstätigkeit in den Jahren des Zweiten Weltkrieges ihren Höchststand: 1943 standen sieben fotografische „Vervielfältigungsapparate“ zur Verfügung, die die Ausgabe von 310.000 „Photokopien“ gestatteten ${ }^{38}$. Konnte die ZWL auf die technische Ausstattung und vielleicht auch die personelle Kompetenz der RWB zurückgreifen? Immerhin steigerte die ZWL schon nach kürzester Anlaufzeit (von ihrem Gründungsjahr 1950 auf 1951) ihren Ausstoß an „Photokopien“ um über $600 \%$, den der „Lesefilme“ um $1.200 \%{ }^{39}$.

War die Arbeit der ZWL primär zur Versorgung der naturwissenschaftlichen und technischen Großforschung gedacht, so partizipierte doch auch das kleine geisteswissenschaftliche Winckelmann-Institut an den Leistungen. Offensichtlich konnten Aufträge ohne institutionelle oder fachliche Einschränkung erteilt

33 Zentralstelle wie Anm. 32, S. 1f.

34 Personalstand von Anfang 1954: Götz wie Anm. 32, S. 2 f.

35 Götz wie Anm. 32, S. 2 f.

36 Kerkhof, Ernst: Reichszentrale für Wissenschaftliche Berichterstattung. In: Jahrbuch der Preußischen Akademie der Wissenschaften (1939), S. 80; ders.: Reichszentrale für Wissenschaftliche Berichterstattung. In: Jahrbuch der Preußischen Akademie der Wissenschaften (1942), S. 80-83; Behrends, Elke: Technisch-wissenschaftliche Dokumentation in Deutschland von 1900 bis 1945. Unter besonderer Berücksichtigung des Verhältnisses von Bibliothek und Dokumentation. Wiesbaden 1995, S. 60-74, 241; dies.: Die Auswirkungen des Boykotts der deutschen Wissenschaft nach dem Ersten Weltkrieg auf das Referatewesen. Die Reichszentrale für Naturwissenschaftliche Berichterstattung. In: Meinel, Christoph (Hg.): Fachschrifttum, Bibliothek und Naturwissenschaft im 19. und 20. Jahrhundert. Wiesbaden 1997, S. 53-66.

37 Kerkhof 1939 wie Anm. 36, S. 81; Behrends 1995 wie Anm. 36, S. 67; Behrends 1997 wie Anm. 36, S. 59.

38 Behrends 1995 wie Anm. 36, S. 68, 241; Behrends 1997 wie Anm. 36, S. 59, 61.

39 Zentralstelle wie Anm. 32, S. 4. 
werden. Angeboten wurden sowohl Mikrofilme als auch Einzelabzüge auf Fotopapier („Photokopien“). Für die Herstellung einer „Ablichtung“ wurden lediglich vier Tage veranschlagt, Auftragsverzögerungen ergaben sich allerdings durch den Beschaffungsaufwand. Die ZWL selber übernahm durch ihre Ermittlungsstelle die Beschaffung der Vorlagen zur Reproduktion und veranschlagte für Vorlagen aus Berlin drei bis vier Wochen, aus der DDR, aus Westdeutschland und dem Ausland drei Monate und mehr. ${ }^{40}$ Die Preispolitik der ZWL zielte auf Kostendeckung, entsprechend konnte das Labor schon nach wenigen Jahren als „rentabler Dienstleistungsbetrieb“ ausgegliedert und dem VEB Agfa Berlin überantwortet werden. ${ }^{41}$ Für einen einzelnen Fotoabzug der Größe DIN A3 wurden 1951 0,95 DM-Ost berechnet, 1953 1,10 DM-Ost ${ }^{42}$. Trotz des hohen Preisniveaus machte das Winckelmann-Institut kurzfristig intensiven Gebrauch von den Dienstleistungen der ZWL. Während die Zentralstelle vor allem aktuelle Westveröffentlichungen verfügbar machen sollte, zielte eine Welle von Aufträgen des Winckelmann-Instituts an das Reprolabor vor allem auf Lückenausgleich im Altbestand, wenn der Buch- oder Antiquariatsmarkt keine Originaldrucke mehr beschaffen konnte. Die ZWL hat diese Aufträge umstandslos umgesetzt. Im Einzelfall ließ man auch druckfrische Publikationen aus dem Westen reproduzieren. Alternativ zu Fotorepros (Abb. 3) wurden - in der Regel bei der ZWL - zahlreiche Mikroverfilmungen (Abb. 4) in Auftrag gegeben, über die ein eigenes Inventar minutiös Auskunft erteilt ${ }^{43}$. Die gehäufte Beauftragung der Reprostelle der ZWL blieb Episode, da sie sich desaströs auf das Institutsbudget auswirkte. Im Rekordbestelljahr 1952 belasteten die Reproduktionen den Bibliotheksetat mit 5.086,70 DM-Ost. So wurden Anfang 1954 zum letzten Mal ZWL-Produkte inventarisiert ${ }^{44}$.

Die Buchzugänge (Ersatzbeschaffungen und Neuerscheinungen) reflektieren auch Besonderheiten des innerdeutschen Buchhandels. Der (West)Berliner Verlag Walter de Gruyter hat einige in (Ost)Berlin durch das Winckelmann-Institut erworbene Eigenpublikationen mit dem Stempel „Das Werk ist für den Gebrauch in der Ostzone [alternativ: DDR] bestimmt [...]“ versehen. Eine der fraglichen Veröffentlichungen ist als im Handel gekauft inventarisiert:

40 Informationen auf dem Auftragsvordruck der ZWL: Universiätsarchiv der Humboldt-Universität: Sektion ÄKuWi, Bereich KLA, Akte Nr. 1131.

41 Götz wie Anm. 32, S. 3.

42 Diverse Unterlagen der ZWL: Universitätsarchiv der Humboldt-Universität: Sektion ÄKuWi, Bereich KLA, Akte Nr. 1131.

43 Winckelmann-Institut der Humboldt-Universität: „Inventarliste über Mikrofilme“.

44 Winckelmann-Institut der Humboldt-Universität: Zugangsbuch der Bibliothek. 


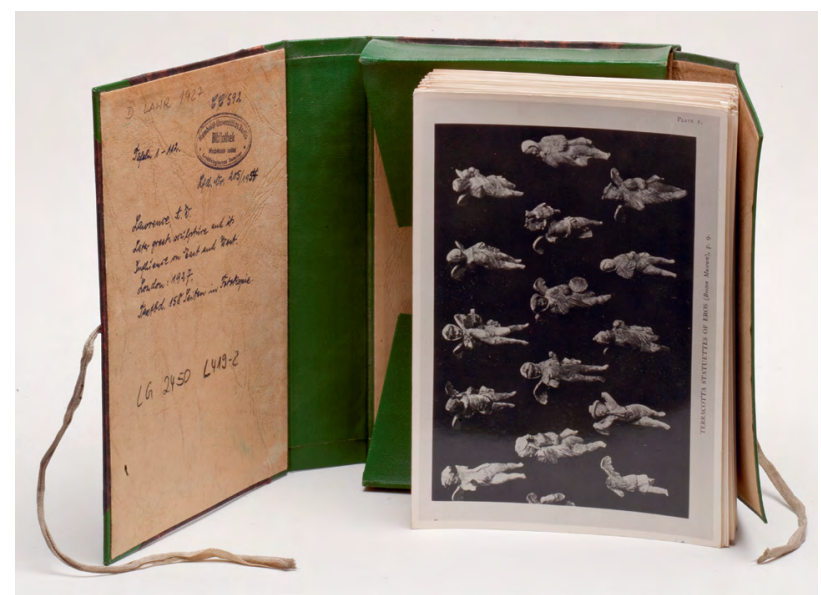

Abb. 3: Arnold W. Lawrence, Later Greek sculpture (1927) als fotografische Reproduktion der Zentralstelle für Wissenschaftliche Literatur, Foto: Antonia Weiße.

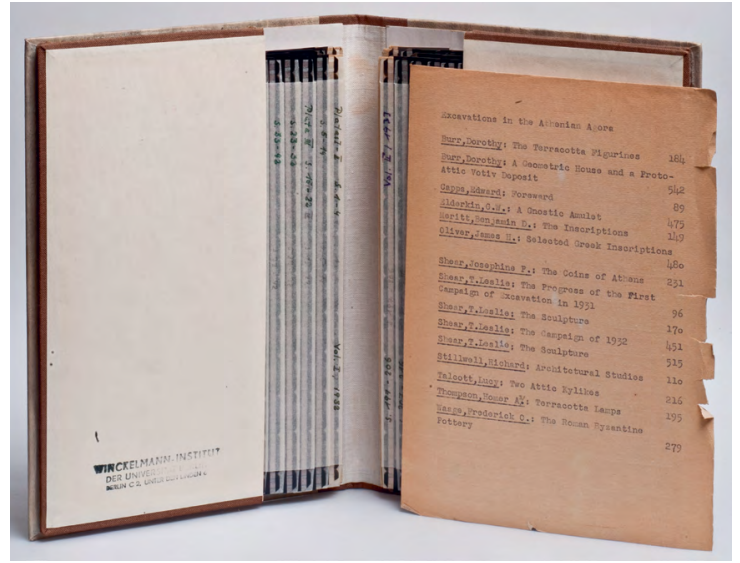

Abb. 4: Ausgrabungsberichte „Excavations in the Athenian Agora“ als Mikroverfilmung der Zentralstelle für Wissenschaftliche Literatur, Foto: Antonia Weiße. 
- Franz Altheim, Römische Geschichte I, Berlin 1948 - Inv. A 1952.11.567 = 1952/43 - Kauf 1952: Harrassowitz, Leipzig - 4,80 M.

In zwei weiteren Fällen sind die Umstände der Anschaffung nicht mehr nachvollziehbar, da die Zugänge lediglich mit Alt-Inventarnummern versehen worden sind:

- Max Ebert (Hg.), Reallexikon der Vorgeschichte, Berlin 1924-1932 - Inv. 7335;

- Jahrbuch des Deutschen Archäologischen Instituts 61/62 (1946/47), Berlin 1949 - Inv. 1950.6704 - 6704 ist die Inventarnummer des Bandes 30 (1915).

Nach schriftlicher Auskunft sind die Umstände dieser Stempelung durch den Verlag nicht mehr zu kontextualisieren - das sollte nicht das letzte Wort gewesen sein.

\section{Stefan Altekamp}

Institut für Archäologie - Winckelmann-Institut

Humboldt-Universität zu Berlin

Unter den Linden 6

10099 Berlin

Deutschland

E-Mail: stefan.altekamp@culture.hu-berlin.de 